\title{
3D Fast Spin-Echo T1 Black-Blood Imaging for the Diagnosis of Cervical Artery Dissection
}

\author{
M. Edjlali, P. Roca, C. Rabrait, O. Naggara, and C. Oppenheim
}

\section{ABSTRACT}

SUMMARY: We performed non-contrast-enhanced 3D fast spin-echo T1 imaging with variable flip angles (CUBE T1) at 3T in 11 patients with CAD. CUBE $T 1$ allowed easy diagnosis of CAD, owing to its comprehensive neck coverage, high spatial resolution enabling multiplanar reformations, fat saturation, and BB effect, the latter also allowing lumen patency to be studied. This sequence may replace 2D axial TIWI for the diagnosis of CAD.

ABBREVIATIONS: $\mathrm{BB}=$ black-blood; $\mathrm{CAD}=$ cervical artery dissection; $\mathrm{IQR}=$ interquartile range

C ervical artery dissection is an increasingly recognized cause of ischemic stroke, particularly in young patients. ${ }^{1}$ Early diagnosis is mandatory because anticoagulation or antithrombotic therapy can help prevent primary or secondary ischemic events. ${ }^{1}$ Axial 2D fat-suppressed T1WI is currently the reference standard to demonstrate the mural hematoma of CAD. Standard MR imaging sensitivity is imperfect, however, and it is not rare for the diagnosis of $\mathrm{CAD}$ to remain presumptive. ${ }^{2} \mathrm{BB}$ sequences with high spatial resolution by using dedicated surface coils may be useful in questionable cases, ${ }^{3}$ but they are time-consuming. Recent technologic innovations allow the acquisition of fat-suppressed 3D FSE T1WI (CUBE T1), with complete neck and head coverage, submillimetric voxels, and acceptable scan duration for clinical practice. ${ }^{4}$ Similar sequences, which differ mainly in the values of the refocusing flip angles, are commercially available (CUBE T1, Vista, and SPACE T1 from GE Healthcare, Milwaukee, Wisconsin; Philips Healthcare, Best, the Netherlands; and Siemens, Erlangen, Germany, respectively). ${ }^{4-6}$ A T1 Vista sequence has already proved useful for the detection of intracranial

Received April 28, 2012; accepted after revision June 12.

From the Department of Neuroradiology, Université Paris-Descartes, Sorbonne Paris Cité, INSERM UMR 894, Centre Hospitalier Sainte-Anne, Paris, France.

M.E. takes full responsibility for the data, the analyses and interpretation, and the conduct of the research and has had access to all of the data; C.O. and M.E. planned the study data collection and identified the patient cohorts; M.E., P.R., C.R., O.N., and C.O. drafted the manuscript. All authors have read and approved the final manuscript and have agreed to conditions noted on the Authorship Agreement Form.

Please address correspondence to Myriam Edjlali, MD, Centre Hospitalier SainteAnne, Service Imagerie Morphologique et Fonctionnelle, 1 Rue Cabanis, 75674 Paris Cedex 14, France; Email: m.edjlali@ch-sainte-anne.fr

http://dx.doi.org/10.3174/ajnr.A3261 dissections. ${ }^{4}$ We hypothesized that CUBE T1 could effectively diagnose $\mathrm{CAD}$ at $3 \mathrm{~T}$.

\section{TECHNIQUE}

CUBE T1 was acquired on a 3T MR imaging scanner (MR 750, GE Healthcare) with a 16-channel array covering the head and neck area with the following parameters: sagittal plane acquisition; FOV, $23 \times 23 \times 16 \mathrm{~cm}^{3}$; TR/TE, $600 / 11.7 \mathrm{~ms}$; echo-train length, 24; initial/minimum flip angle, $120^{\circ} / 25^{\circ}$; bandwidth, $62.5 \mathrm{kHz}$; matrix, $288 \times 288 \times 160$ interpolated to $512 \times 512 \times 320$; voxel size of $0.4 \times 0.4 \times 0.5 \mathrm{~mm}^{3}$ after interpolation. Parallel acceleration was applied along the phase direction (Autocalibrating Reconstruction for Cartesian Sampling, factor of 2). Variable refocusing flip angles ${ }^{5}$ were used to compensate for the signal decay inherent in the long echo-train length used to reduce scanning time in fast spin-echo sequences. An inferior outer volume suppression pulse was applied to limit inflow effects, and a fat-saturation pulse was added to reduce the signal from subcutaneous and facial fat. The craniocaudal coverage ranged from above the clavicles to the vertex for a total scanning time of 7 minutes 49 seconds.

Eleven consecutive patients with a definite diagnosis of recent ( $<1$ month from the onset of symptoms) CAD were imaged with a CUBE T1 sequence between July 2011 and January 2012. CAD diagnosis was based on comprehensive clinical and imaging investigations available at hospital discharge, including cervical sonography, brain MR imaging (except for CUBE T1), contrastenhanced MRA, and standard axial fat-suppressed T1WI $(n=9)$. All patients demonstrated a mural hematoma on at least 1 of the different imaging investigations. On axial fat-suppressed T1WI, the hematoma was hyperintense in 6 cases, doubtful in 2, and not visible in 1 case.

Two readers (M.E., C.O.), aware that all patients had CAD but 

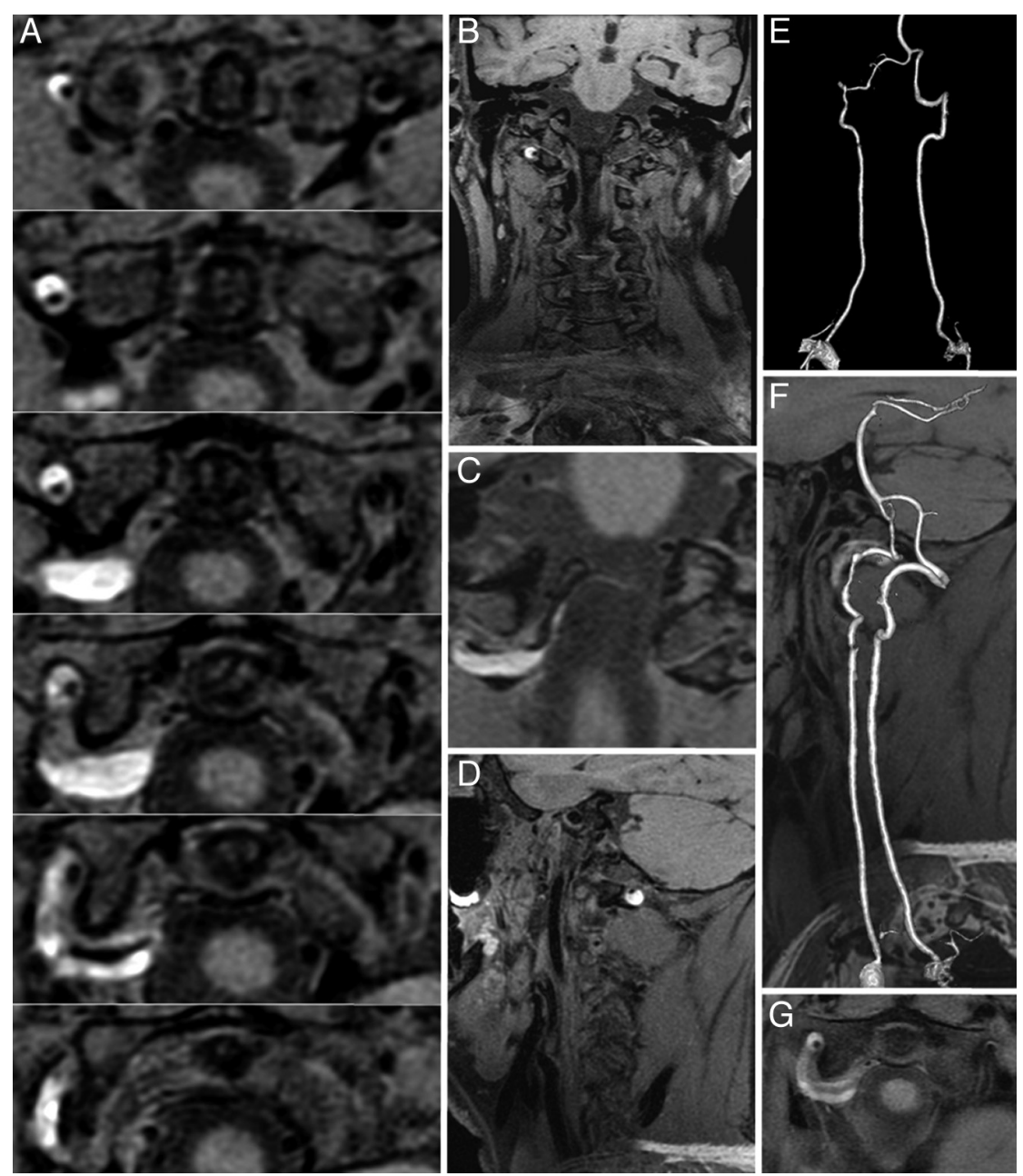

FIG 1. $A$, CUBE $T 1$ consecutive axial views (thickness, $0.5 \mathrm{~mm}$ ) covering the $\mathrm{V} 3$ vertebral segment. A bright crescentic signal (mural hematoma) surrounds the dark arterial lumen of the right vertebral artery. $B$ and $D$, CUBE T1 coronal and sagittal views show the head and neck coverage and the mural hematoma. $C$, CUBE T1 oblique view centered on the V3-V4 junction shows that the vertebral dissection thinly extends intradurally. E, Contrast-enhanced MRA (CE-MRA) shows irregularity without stenosis of the right $\mathrm{V} 3$ segment lumen. $F$, CUBE T1 sagittal view fused with CE-MRA shows the bright T1 hematoma surrounding an irregular arterial lumen. G, Standard axial T1 BB image with fat saturation shows that the hematoma is also seen surrounding the right V3 segment. blinded to the arterial location, independently reviewed CUBE T1 images to search for a mural hematoma. They were instructed to conclude "definite mural hematoma" when hyperintense wall thickening was present on 3 orthogonal reformations, "possible CAD" in the case of wall thickening isointense to the sternocleidomastoid muscle, or "wall normality." In the case of possible CAD, a second CUBE T1 was performed 3 days later.

\section{RESULTS}

Among the 11 patients with CAD (10 men) with a median (IQR) age of 42.0 years (range, $36-47$ years), there were 5 carotid CADs in 5 patients, 7 vertebral CADs in 6 patients, and 32 undissected arteries. Seven patients had local symptoms, 4 had Horner syndrome, and 4 had an ischemic lesion ipsilateral to the CAD. The median delay from onset to CUBE T1 MRI was 5 days with a range of 1-24 days.

On CUBE T1, a definite mural hematoma (Figs 1 and 2) was seen by both readers in 10 (all 5 carotid CADs and 5 patients with 6 vertebral CADs) of the 11 patients with CAD. In 1 patient, a mural hematoma of the V3 vertebral segment was visible on sonographic examination but was recorded as possible by both readers on CUBE T1 obtained 48 hours after CAD onset. This latter patient was rescanned 5 days after onset, and the mural hematoma was then considered definite for both readers on CUBE T1.

CUBE T1 findings were considered normal in all 32 non-CAD arteries by both readers. An intradural extension was reported to be visible in 2 vertebral CADs by both readers.

In the first 5 patients, CUBE T1 was acquired without a high dielectric constant pad. Because of major signal loss ${ }^{7}$ at the cervical level (Fig 3), observed in 2 cases, we repeated the MR imaging during the same imaging session after having placed a dielectric pad filled with a dilute manganese chloride solution under the patient's neck. This improved image quality, and subsequently all MR imaging acquisitions were performed by using a dielectric pad.

\section{DISCUSSION}

CUBE T1 allows easy visualization of mural hematoma in CAD. The contrast between the hyper-T1 signal of the hematoma and the surrounding tissues, associated with a high spatial resolution, enables the reader to have several sections with good contrast covering the dissected arterial segment, even in the case of localized CAD (Fig 1). CUBE T1 offers several advantages over the fat-suppressed axial T1 sequences commonly used for the detection of mural hematoma: 1) absence of inflow artifacts due to the $3 \mathrm{D}$ acquisition and the use of an outer volume-suppression pulse; 2) a non-cardiac-gated BB effect due to the presence of different intravoxel velocities in blood vessels, causing a phase dispersion and resulting in signal loss, a phenomenon amplified with the use of long echo trains and low refocusing flip angles ${ }^{5}$; 3 ) homoge- 

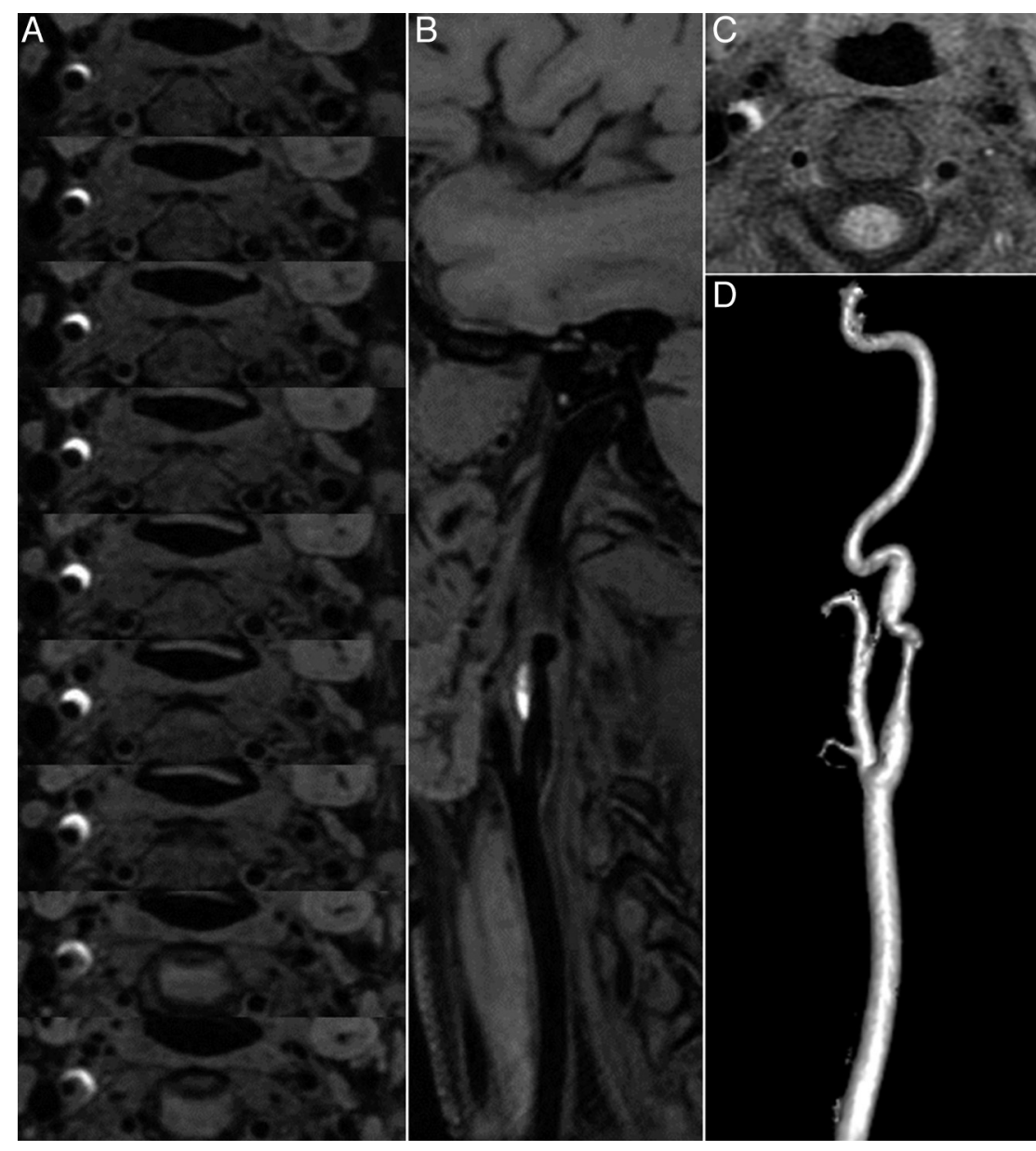

FIG 2. $A$, CUBE $T 1$ consecutive axial views (thickness, $0.5 \mathrm{~mm}$ ) covering the dissected right carotid artery show a bright crescentic signal surrounding the dark arterial lumen of the internal carotid artery. $B$, CUBE T1 oblique view oriented along the common carotid axis shows the dark lumen of the artery and the bright crescent signal of the hematoma. $C$, Standard axial Tl BB image with fat saturation shows that the hematoma is also seen surrounding the right internal carotid artery, but only on 2 sections. D, Contrast-enhanced MRA shows irregularity with stenosis of the suprabulbar internal carotid artery.
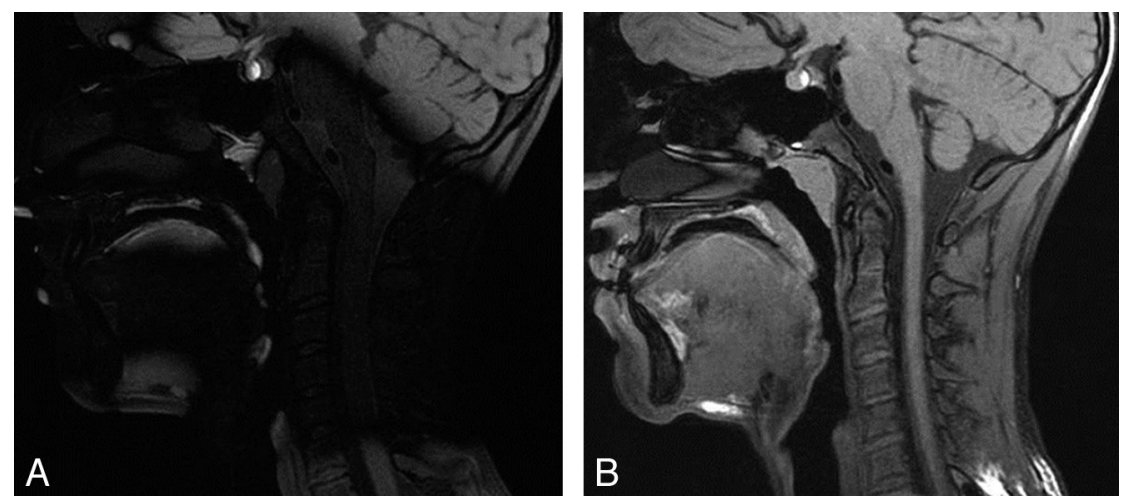

FIG 3. $3 D T 1$ sagittal views of $2 M R$ imaging acquisitions in the same patient without $(A)$ and with $(B)$ a dielectric pad.

neous fat suppression; 4) a 3D acquisition with submillimetric and almost isotropic voxels, allowing multiplanar reformations; and 5) a large field of view in the z-axis for comprehensive head and neck coverage. These technical improvements support the use of CUBE T1 as a first-line diagnostic tool for CAD and potentially overcome the limitations of high-resolution $\mathrm{BB}$ sequences, which have previously required the use of dedicated surface coils and have focused on the abnormal segment of the dissected artery.

Dielectric artifacts ${ }^{7}$ may lower the image quality of CUBE T1 by disrupting the optimal set of variable flip angles, thus inducing a loss of signal in affected zones. This effect was counterbalanced by the use of a dielectric constant pad located under the patient's neck. Selective shimming focused on the region of interest could also be used to provide better local field homogeneity.
Our study has several limitations. First, the sample size was small, and only 3 patients were imaged within 48 hours after the onset of symptoms. Because of the small number of patients, we did not compare the diagnostic confidence of CUBE T1 for vertebral-versus-carotid CAD. Second, because the readers were aware that all patients had CAD, we could not assess the sensitivity of this technique compared with standard methods for diagnosing mural hematomas. Larger cohorts of patients will be necessary to assess the sensitivity and specificity of CUBE T1 for CAD diagnosis.

\section{CONCLUSIONS}

CUBE T1 allowed easy identification of the mural hematoma of $\mathrm{CAD}$, owing to its comprehensive neck coverage, high spatial res-

AJNR Am J Neuroradiol 34:E103-E06 Sep 2014 www.ajnr.org

E105 
olution enabling multiplanar reformations, fat saturation, and BB effect. This sequence may be a substitute for $2 \mathrm{D}$ axial sequences in the diagnosis of CAD.

Disclosures: Myriam Edjlali—RELATED: Support for Travel to Meetings for the Study or Other Purposes: GE Healthcare, Comments: 2012 ISMRM Congress, Pauline Roca-UNRELATED: Employment: GE Healthcare, ${ }^{*}$ Comments: I hold a postdoctoral position at Sainte-Anne Hospital, funded by GE Healthcare, Travel/Accommodations/Meeting Expenses Unrelated to Activities Listed: GE Healthcare, Comments: ISMRM 2012 Congress (Melbourne), and SFRMBM 2012 French congress (Marseille). Cécile Rabrait-UNRELATED: Employment: GE Healthcare, Comments: I work as a Clinical Scientist for GE Healthcare. Catherine Oppenheim-UNRELATED: Payment for Development of Education Presentations: GE Healthcare, Comments: Two educational presentations in 2011 (cumulative amount $<\$ 5000$ ). *Money paid to the institution.

\section{REFERENCES}

1. Debette S, Leys D. Cervical-artery dissections: Predisposing factors, diagnosis, and outcome. Lancet Neurol 2009;8:668-78
2. Anzalone N, Cadioli M. Vertebral artery dissection: looking for the ideal study protocol. AJNR Am J Neuroradiol 2011;32:E91, author reply E92

3. Naggara $\mathrm{O}$, Louillet $\mathrm{F}$, Touze $\mathrm{E}$, et al. Added value of high-resolution MR imaging in the diagnosis of vertebral artery dissection. AJNR Am J Neuroradiol 2010;31:1707-12

4. Takemoto $\mathrm{K}$, Takano $\mathrm{K}$, Abe $\mathrm{H}$, et al. The new MRI modalities "BPAS and VISTA" for the diagnosis of VA dissection. Acta Neurochir Suppl 2011;112:59-65

5. Busse RF, Brau AC, Vu A, et al. Effects of refocusing flip angle modulation and view ordering in 3D fast spin echo. Magn Reson Med 2008;60:640-49

6. Kato Y, Higano S, Tamura H, et al. Usefulness of contrast-enhanced T1-weighted sampling perfection with application-optimized contrasts by using different flip angle evolutions in detection of small brain metastasis at 3T MR imaging: comparison with magnetization-prepared rapid acquisition of gradient echo imaging. AJNR Am J Neuroradiol 2009;30:923-29

7. Bernstein MA, Huston J 3rd, Ward HA. Imaging artifacts at 3.0T. $J$ Magn Reson Imaging 2006;24:735-46 